\title{
Gene Amplification
}

National Human Genome Research Institute (NHGRI)

\section{Source}

National Human Genome Research Institute (NHGRI). Gene Amplification.

Gene amplification is an increase in the number of copies of a gene sequence. Cancer cells sometimes produce multiple copies of genes in response to signals from other cells or their environment. The term also can refer to polymerase chain reaction (PCR), a laboratory technique that is used by scientists to amplify gene sequences in a test tube. 